\title{
Emulation-based stabilisation of networked control systems over WirelessHART
}

\author{
Alejandro I. Maass, Dragan Nešić, Romain Postoyan, Peter M. Dower and Vineeth S. Varma
}

\begin{abstract}
We study the emulation-based stabilisation of nonlinear networked control systems (NCSs) implemented over WirelessHART (WH). WH is a communication protocol widely used in process instrumentation. It is characterised by its multi-hop structure, slotted communication cycles, and simultaneous transmission over different frequencies. To capture most functionalities of WH, faithful models are needed. We propose a hybrid control-oriented model of WH-NCSs that includes the key features of the network. We then follow an emulation approach to stabilise the NCS. We show that, under reasonable assumptions on the scheduling protocol, stability is preserved when the controller is implemented over the network with sufficiently frequent data transmission. We then explain how to schedule transmissions over the hops to satisfy those assumptions.
\end{abstract}

\section{INTRODUCTION}

A control system where the plant and the controller communicate over a digital network is called a networked control system (NCS). Numerous works in the literature deal with constraints induced by the network, see e.g., [1], [2]. While these results capture the essential effects that the network has on the closed-loop system, it remains unclear how these can be applied to specific physical networks such as WirelessHART (WH), FlexRay, etc. We are motivated to develop results tailored to NCSs implemented over WirelessHART, a recent wireless communication standard used in process control [3]. WH is a mesh network, which utilises field devices in a multi-hop fashion. Such devices act as buffers to forward data packets. In addition, communications are precisely scheduled using time division multiple access (TDMA), and all its frequency channels may be simultaneously used to transmit.

Existing results concerning WH include optimal link scheduling [4], [5], controller-communications co-design [6], [7], and modelling and analysis [8], [9]. Most of the aforementioned results model the network as a directed graph with a set of nodes and links. Also, if packet losses between nodes take place, then these are modelled as a Markov chain. Linear and discrete-time plant/controller models are considered, together with equidistant transmission instants. Such assumptions may be hard to implement in real WH networks,

This work was supported by the ARC Discovery Scheme, grant number DP170104099, and by the ANR project COMPACS, ANR-13-BS03-004-02.

A.I. Maass, D. Nešić and P.M. Dower are with the Department of Electrical and Electronic Engineering, The University of Melbourne, Parkville, 3010, Victoria, Australia. amaass Q student . unimelb.edu . au, dnesic@unimelb.edu.au, pdower@unimelb.edu.au.

R. Postoyan and V.S. Varma are with the Université de Lorraine, CRAN, UMR 7039 and the CNRS, CRAN, UMR 7039, France. romain.postoyanduniv-lorraine.fr, vineeth. satheeskumar-varma@univ-lorraine.fr. where extra features need to be taken into consideration e.g., TDMA communications, time-varying transmission instants and scheduling. The first purpose of this study is to propose a model, which is able to capture these features. To that end, we adopt the modelling formalism of hybrid control systems [10]. It serves as a starting point for high-fidelity and controloriented modelling of NCSs implemented on real physical networks. For example, recent works in [11], [12] use this formalism to obtain a high-fidelity hybrid model of a network used in automotive control called FlexRay.

In this paper, we present a control-oriented hybrid model of NCSs over WH that captures inter-sampling behaviour, time-varying transmission instants, field device dynamics, and nonlinear plant and controller. We then use this model to analyse stability in the case where the controller is designed by emulation. The main idea of emulation is to first design a controller that stabilises the plant in the absence of the network. Then, the controller is implemented over the network and it is shown that the stability of the system is preserved, see e.g., [13]-[15]. In particular, stability is preserved under reasonable conditions on the scheduling protocols, and sufficiently frequent transmissions, measured by the maximum allowable transmission interval (MATI). Compared to the models in [13]-[15], our hybrid model is adapted to cope with the specificity of the WH standard, i.e., buffer dynamics and time-slotted communication cycles. Despite these differences, stability results in [14] can still be almost directly applied provided the scheduling protocols, that governs transmissions between field devices, are uniformly globally exponential stable (UGES). We present a general class of TDMA scheduling protocols for which these assumptions hold and we provide relevant examples that belong to this class. In particular, we show that the use of multiple simultaneous transmissions over different frequencies, can be exploited to improve MATI bounds. These results can also be used to design scheduling policies for field devices in WirelessHART networks.

In our preliminary work [16], we obtained a first hybrid model for WH-NCSs. However, the buffer states of field devices were not included in the model; a restriction that permits only one transmission per timeslot per frequency was omitted; and scheduling of field devices along the network radio frequencies was another missing feature.

\section{Preliminaries}

Denote by $\mathbb{R}$ the set of real numbers. Let $\mathbb{R}_{\geq 0} \doteq[0, \infty)$, $\mathbb{Z}_{\geq 0} \doteq\{0,1,2, \ldots\}$, and $\mathbb{N} \doteq\{1,2,3, \ldots\}$. For vector arguments, $|\cdot|$ denotes the Euclidean norm. The same 


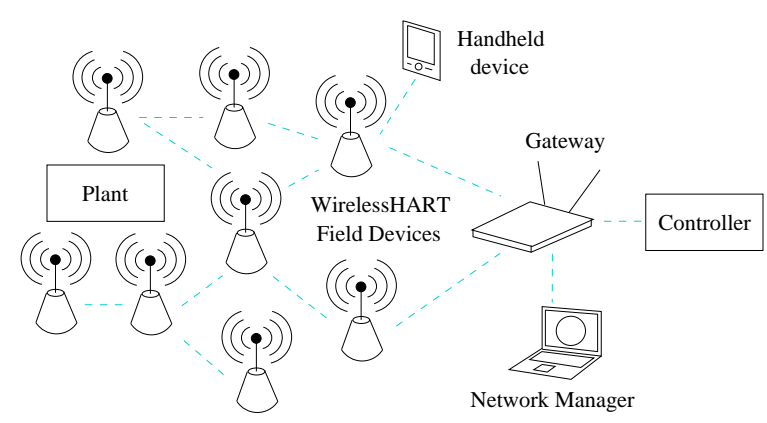

Fig. 1. WirelessHART architecture.

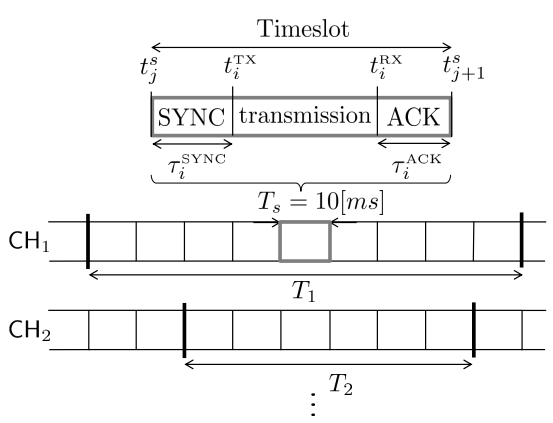

Fig. 2. WirelessHART superframe table. notation is used to denote the induced 2-norm of a matrix. For simplicity, we use $(x, y) \doteq\left[\begin{array}{ll}x^{T} & y^{T}\end{array}\right]^{T} \in \mathbb{R}^{n+m}$. A function $\alpha: \mathbb{R}_{\geq 0} \rightarrow \mathbb{R}_{\geq 0}$ is of class $\mathcal{K}$ if it is continuous, zero at zero and strictly increasing. It is of class $\mathcal{K}_{\infty}$ if it is of class $\mathcal{K}$ and unbounded. A function $\beta: \mathbb{R}_{\geq 0} \times \mathbb{R}_{\geq 0} \rightarrow \mathbb{R}_{\geq 0}$ is of class $\mathcal{K} \mathcal{L}$ if $\beta(\cdot, t)$ is of class $\mathcal{K}$ for each $t \geq 0$, and if $\beta(s, \cdot)$ is continuous, nonincreasing and satisfies $\lim _{t \rightarrow \infty} \beta(s, t)=0$ for each $s \geq 0$. Given $t \in \mathbb{R}$ and a piecewise continuous function $f: \mathbb{R} \rightarrow \mathbb{R}^{n}$, we use the notation $f\left(t^{+}\right) \doteq \lim _{s \rightarrow t, s>t} f(s)$. We define $\mathbf{1}_{A}$ as the function $\mathbf{1}_{A}: \mathbb{N} \rightarrow\{0,1\}$ such that $\mathbf{1}_{A}(i)=1$ for $i \in A$, and $\mathbf{1}_{A}(i)=0$ for $i \notin A$.

\section{WIRELESSHART NETWORK}

A description of WirelessHART is provided as a prelude to the model development to follow in Section IV.

\section{A. Communication features}

The general architecture of a WH network is shown in Fig. 1. It consists of an interconnection of basic components including field devices that communicate with the plant process (e.g. sensor/actuators), handheld devices to run diagnostics, gateways that enable communications between host applications and field devices, and a network manager responsible of scheduling. WH is based on the IEEE 802.15.4-2006 physical layer and operates in the $2.4 \mathrm{GHz}$ ISM radio band with a maximum data rate of $250 \mathrm{kbits} / \mathrm{s}$ over 15 frequency division multiplexed channels. In the data link layer, WH defines a slotted TDMA technology. That is, each frequency channel is subdivided into timeslots in which an assigned field device is allowed to transmit. WH networks also support multiple access timeslots, however, in this work we restrict our attention to TDMA communications as in [16].

\section{B. TDMA superframe structure}

All communications in a WH network are defined with respect to a superframe. A superframe is an a priori fixed period of time $T_{h}>0, h=1, \ldots, 15$, contiguous in real time with other superframes, that is divided into a sequence of timeslots as depicted in Fig. 2. Field devices are scheduled to transmit in the superframe, and each one of the 15 channels may have a different superframe. The set of superframes along frequency channels is called a superframe table. Note that superframe lengths $T_{h}$ are flexible and depend on scheduling. Each timeslot is strictly $T_{s}=10 \mathrm{~ms}$ in duration. Within this timeslot, a complete single data packet and its corresponding acknowledgement are transmitted between two field devices, see Fig. 2. The transmission delay is denoted by $t_{i}^{\mathrm{RX}}-t_{i}^{\mathrm{TX}}$ and it depends on the packet size. Acknowledge time is denoted by $\tau_{i}^{\text {ACK }}$. In order to have effective TDMA communications, all devices need to be synchronized, see $\tau_{i}^{\text {SYNC }}$ in Fig. 2. For simplicity, and similar to [16], the following additional assumption is adopted.

Assumption 1: The following holds.

(a) Packets are transmitted instantaneously in every timeslot, i.e., $t_{i} \doteq t_{i}^{\mathrm{TX}}=t_{i}^{\mathrm{RX}}$ for all $i \in \mathbb{N}$. We refer to $t_{i}$ as transmission instant.

(b) Acknowledgement time is negligible in each timeslot, i.e., $\tau_{i}^{\mathrm{ACK}}=0$ for all $i \in \mathbb{N}$.

(c) One successful transmission between devices occurs within each timeslot, per channel.

(d) Transmissions across all channels are synchronized. We define $\varepsilon \doteq \inf _{i \in \mathbb{N}} \tau_{i}^{\text {SYNC }}$ and assume that $\varepsilon>0$.

As foreshadowed in the introduction, in this paper we will parametrize our model with the so-called maximal allowable transmission interval (MATI) [15], which we denote as $\tau_{\text {MATI }}>0$. In other words, a packet must be transmitted at most in $\tau_{\text {MATI }}$ seconds after the previous packet transmission to preserve stability. Let us introduce the time instants $t_{j}^{s}$ for $j \in \mathbb{Z}_{\geq 0}$ (see Fig. 2), that are such that $t_{j}^{s}=j T_{s}$. The following lemma follows directly from Assumption 1 and the latter definitions.

Lemma 1: If Assumption 1 holds, given any $i \in \mathbb{N}$ there exists $j \in \mathbb{Z}_{\geq 0}$, such that $t_{j}^{s}+\varepsilon \leq t_{i} \leq t_{j+1}^{s}$, where $\varepsilon>0$ comes from Assumption 1.

The next corollary comes from Lemma 1 and Assumption 1.

Corollary 1: The transmission instants $t_{i}$ in the WH network, satisfy $\varepsilon \leq t_{i+1}-t_{i} \leq \tau_{\text {MATI }} \leq 2 T_{s}-\varepsilon$ for all $i \in \mathbb{N}$.

Lemma 1 captures the fact that there is only one transmission within a timeslot on a given frequency. Corollary 1 captures the fact that a packet must be transmitted at most in $\tau_{\text {MATI }}$ seconds, which cannot be larger than $2 T_{s}-\varepsilon$.

\section{MOdel OF A WH-NCS}

Consider the NCS shown in Fig. 3 with

$$
\dot{x}_{p}=f_{p}\left(x_{p}, \hat{u}\right), \quad y=g_{p}\left(x_{p}\right),
$$

as the plant model, where $x_{p} \in \mathbb{R}^{n_{p}}$ is the state, $\hat{u} \in \mathbb{R}^{n_{u}}$ is the control signal received by the plant, $y \in \mathbb{R}^{n_{y}}$ is the plant output, and $n_{p}, n_{u}, n_{y} \in \mathbb{N}$. The controller is given by

$$
\dot{x}_{c}=f_{c}\left(x_{c}, \hat{y}\right), \quad u=g_{c}\left(x_{c}\right)
$$




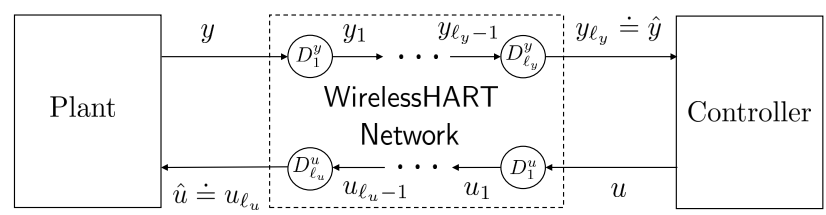

Fig. 3. NCS implemented over a WH network with $\ell_{y}$ field devices in the plant-to-controller link and $\ell_{u}$ field devices in the controller-to-plant link.

where $x_{c} \in \mathbb{R}^{n_{c}}$ is the state of the controller, $\hat{y} \in \mathbb{R}^{n_{y}}$ is the plant output received by the controller, $u \in \mathbb{R}^{n_{u}}$ is the control signal, and $n_{c} \in \mathbb{N}$. The functions $f_{p}, f_{c}$ are assumed to be continuous and $g_{p}, g_{c}$ are assumed to be continuously differentiable.

We model the WH network as in Fig. 3. That is, we consider $\ell_{y} \in \mathbb{Z}_{\geq 0}$ field devices interconnected in the plantto-controller path (we refer to it as $y$-path) and $\ell_{u} \in \mathbb{Z}_{\geq 0}$ in the controller-to-plant path (we refer to it as $u$-path). We label the field devices as $D_{\alpha}^{y}$ and $D_{\beta}^{u}$, where $\alpha=$ $1, \ldots, \ell_{y}$ and $\beta=1, \ldots, \ell_{u}$, see Fig. 3. According to $\mathrm{WH}$ specifications, each field device acts as a router for data from/to neighbouring field devices. Therefore, we model field devices as buffers, for which we introduce buffer state variables denoted by $b_{\alpha}^{y}$ and $b_{\beta}^{u}$, for field devices in the $y$ path and $u$-path, respectively. In particular, we model the reception and transmission behaviour of field devices in the $y$-path as follows ( $u$-path devices are modelled identically)

$$
\begin{aligned}
\dot{y}_{\alpha}(t) & =0, & \dot{b}_{\alpha}^{y}(t) & =0, \\
y_{\alpha}\left(t_{i}^{+}\right) & =b_{\alpha}^{y}\left(t_{i}\right), & b_{\alpha}^{y}\left(t_{i}^{+}\right) & =b_{\alpha}^{y}\left(t_{i}\right), \\
y_{\alpha+1}\left(t_{i}^{+}\right) & =y_{\alpha+1}\left(t_{i}\right), & b_{\alpha+1}^{y}\left(t_{i}^{+}\right) & =y_{\alpha}\left(t_{i}^{+}\right),
\end{aligned}
$$

for all $\alpha=1, \ldots, \ell_{y}, t \in\left[t_{i}, t_{i+1}\right]$, and $i \in \mathbb{N}$. Note that for $\alpha=\ell_{y}$, equation (3c) does not apply, and (3b) represents device $D_{\ell_{y}}^{y}$ transmitting its buffer content to the controller.

To simplify the stability analysis that follows, we define the network induced errors for the $y$-path and $u$-path as

$$
\begin{gathered}
\zeta^{y} \doteq\left(b_{1}^{y}-y, b_{2}^{y}-y_{1}, \cdots, b_{\ell_{y}}^{y}-y_{\ell_{y}-1},\right. \\
\left.y_{1}-b_{1}^{y}, y_{2}-b_{2}^{y}, \cdots, y_{\ell_{y}}-b_{\ell_{y}}^{y}\right), \\
\zeta^{u} \doteq\left(b_{1}^{u}-u, b_{2}^{u}-u_{1}, \cdots, b_{\ell_{u}}^{u}-u_{\ell_{u}-1},\right. \\
\left.u_{1}-b_{1}^{u}, u_{2}-b_{2}^{u}, \cdots, u_{\ell_{u}}-b_{\ell_{u}}^{u}\right) .
\end{gathered}
$$

The first $\ell_{y}$ components of $\zeta^{y}$ (resp. $\ell_{u}$ components of $\left.\zeta^{u}\right)$, are related to the buffer update during reception. The remaining $\ell_{y}$ components of $\zeta^{y}$ (resp. $\ell_{u}$ components of $\zeta^{u}$ ), are related to the transmission of such buffer value through their output. In particular, we will reset to zero these errors to model reception and transmission. This is a major difference with previous models like [13]-[15], in which the network induced error is given by $e \doteq(\hat{y}-y, \hat{u}-u)$ (i.e., no specific network is considered, and the possible buffer dynamics are ignored). Define also the augmented states $x \doteq\left(x_{p}, x_{c}\right)$, and $\zeta \doteq\left(\zeta^{y}, \zeta^{u}\right)$, where $x \in \mathbb{R}^{n_{x}}, \zeta \in \mathbb{R}^{n_{\zeta}}, n_{x} \doteq n_{p}+n_{c}$, $n_{\zeta} \doteq n_{\zeta^{y}}+n_{\zeta^{u}}, n_{\zeta^{y}} \doteq 2 \ell_{y} n_{y}$, and $n_{\zeta^{u}} \doteq 2 \ell_{u} n_{u}$.

By using (1)-(4), we present an impulsive model, for the block diagram in Fig. 3 between and at transmission instants.
In particular, for all $t \in\left[t_{i}, t_{i+1}\right]$,

$$
\begin{aligned}
(\dot{x}(t), \dot{\zeta}(t)) & =(f(x(t), \zeta(t)), g(x(t), \zeta(t))), \\
\left(x\left(t_{i}^{+}\right), \zeta\left(t_{i}^{+}\right)\right) & =\left(x\left(t_{i}\right), h\left(i, \zeta\left(t_{i}\right)\right)\right), \\
\left(x\left(t_{j}^{s+}\right), \zeta\left(t_{j}^{s+}\right)\right) & =\left(x\left(t_{j}^{s}\right), \zeta\left(t_{j}^{s}\right)\right),
\end{aligned}
$$

where $f$ and $g$ are obtained by direct calculations from (1), (2) and (4), and where $h$ is a function that decides when field devices are scheduled to transmit. We refer to $\zeta\left(t_{i}^{+}\right)=$ $h\left(i, \zeta\left(t_{i}\right)\right)$ as the protocol equation.

We now embed the impulsive model (5) into a hybrid system so that we can resort to the analytical tools of [10]. To that end, we introduce a clock variable $\tau \in \mathbb{R}_{\geq 0}$, which represents the time elapsed since the last transmission. We also need a clock variable $\tau_{s} \in \mathbb{R}_{\geq 0}$, which corresponds to the time elapsed on each timeslot. Let us introduce $\kappa \in \mathbb{Z}_{\geq 0}$, which counts the number of transmissions. Also, we need another counter $q \in\{0,1\}$ to limit transmissions within the timeslot to one. Another difference with prior work in [13], [14], is the inclusion of the clock $\tau_{s}$ and the counter $q$ to cope with the specificity of WH.

Define the hybrid state $\xi \doteq\left(x, \zeta, \tau, \kappa, \tau_{s}, q\right)$. In that way, by using (5), Lemma 1 and Corollary 1, we present the following hybrid model for NCSs implemented over WH networks

$$
\begin{aligned}
\dot{\xi} & =\mathcal{F}(\xi), \quad \xi \in C, \\
\xi^{+} & =\mathcal{G}(\xi), \quad \xi \in D,
\end{aligned}
$$

where the flow and jump sets are given by

$$
\begin{aligned}
C & \doteq \mathbb{R}^{n_{x}} \times \mathbb{R}^{n_{\zeta}} \times\left[0, \tau_{\text {MATI }}\right] \times \mathbb{Z}_{\geq 0} \times\left[0, T_{s}\right] \times\{0,1\}, \\
D & \doteq D^{\text {trans }} \cup D^{\text {slot }}, \\
D^{\text {trans }} & \doteq \mathbb{R}^{n_{x}} \times \mathbb{R}^{n_{e}} \times \mathbb{R}_{\geq 0} \times \mathbb{Z}_{\geq 0} \times\left[\varepsilon, T_{s}\right] \times\{0\}, \\
D^{\text {slot }} & \doteq \mathbb{R}^{n_{x}} \times \mathbb{R}^{n_{e}} \times \mathbb{R}_{\geq 0} \times \mathbb{Z}_{\geq 0} \times\left\{T_{s}\right\} \times\{1\},
\end{aligned}
$$

and where the mappings $\mathcal{F}$ and $\mathcal{G}$ are defined as

$$
\begin{aligned}
& \mathcal{F}(\xi) \doteq(f(x, \zeta), g(x, \zeta), 1,0,1,0), \\
& \mathcal{G}(\xi) \doteq \begin{cases}\left(x, h(\kappa, \zeta), 0, \kappa+1, \tau_{s}, 1-q\right), & \xi \in D^{\text {trans }}, \\
(x, \zeta, \tau, \kappa, 0,0), & \xi \in D^{\text {slot }} .\end{cases}
\end{aligned}
$$

The hybrid model (6) is subject to two different jumps, namely transmission jumps (when $\xi \in D^{\text {trans }}$ ) and timeslot switching jumps (when $\xi \in D^{\text {slot }}$ ). If a transmission occurred within the timeslot, then $q^{+}=1$, and the next transmission cannot happen before the next timeslot (i.e., before $\tau_{s}=T_{s}$ ). Therefore, at timeslot switching jumps $q^{+}=0$, and the next transmission is allowed to happen after $\varepsilon$ time units.

\section{STABility RESUlTS}

Although system (6) differs from the previous model considered in [14], we can resort to the same tools to ensure its stability. We make the following assumption on (6).

Assumption 2: There exist a function $W: \mathbb{Z}_{\geq 0} \times \mathbb{R}^{n_{e}} \rightarrow$ $\mathbb{R}_{\geq 0}$ that is locally Lipschitz in its second argument, a continuous function $H: \mathbb{R}^{n_{x}} \rightarrow \mathbb{R}_{\geq 0}$, real numbers $\lambda \in$ 
$(0,1), L \geq 0, \gamma>0$, and functions $\underline{\alpha}_{W}, \bar{\alpha}_{W} \in \mathcal{K}_{\infty}$, such that, for all $\kappa \in \mathbb{Z}_{\geq 0}$ and $\zeta \in \mathbb{R}^{n_{\zeta}}$,

$$
\begin{aligned}
\underline{\alpha}_{W}(|\zeta|) & \leq W(\kappa, \zeta) \leq \bar{\alpha}_{W}(|\zeta|), \\
W(\kappa+1, h(\kappa, \zeta)) & \leq \lambda W(\kappa, \zeta),
\end{aligned}
$$

and for all $\kappa \in \mathbb{Z}_{\geq 0}, x \in \mathbb{R}^{n_{x}}$ and almost all $\zeta \in \mathbb{R}^{n_{\zeta}}$,

$$
\left\langle\frac{\partial W(\kappa, \zeta)}{\partial \zeta}, g(x, \zeta)\right\rangle \leq L W(\kappa, \zeta)+H(x),
$$

in which $g$ is as per (5). Moreover, there exists a locally Lipschitz, positive definite, radially unbounded function $V$ : $\mathbb{R}^{n_{x}} \rightarrow \mathbb{R}_{\geq 0}$, and a continuous, positive definite function $\varrho$, such that, for all $\zeta \in \mathbb{R}^{n_{\zeta}}$, all $\kappa \in \mathbb{Z}_{\geq 0}$, and almost all $x \in \mathbb{R}^{n_{x}}$,

$$
\begin{array}{r}
\langle\nabla V(x), f(x, \zeta)\rangle \leq-\varrho(|x|)-\varrho(W(\kappa, \zeta)) \\
-H(x)^{2}+\gamma^{2} W(\kappa, \zeta)^{2},
\end{array}
$$

where $f$ is as per (5).

Condition (7) is related to the UGES of the scheduling protocol [13]. The difference with previous work is that now $W$ depends on $\zeta$, which involves the buffer state variables related to field devices in a WH network. We show in Section VI that several configurations implementable in WH lead to maps $h$ which verify (7). Condition (8) corresponds to an exponential growth condition on the $\zeta$-system. It is often satisfied when $W$ is globally Lipschitz in $\zeta$, which is the case for the examples in Section VI, and when $g$ grows linearly for instance. In particular, it suffices to show that: there exists $L_{1} \geq 0$ such that for almost all $\zeta \in \mathbb{R}^{n_{\zeta}}$ and $i \in \mathbb{N}$, we have

$$
\left|\frac{\partial W(i, \zeta)}{\partial \zeta}\right| \leq L_{1}
$$

and that there exists $L_{2} \geq 0$ such that $|g(x, \zeta)| \leq L_{2}(|\zeta|+$ $|x|)$. Indeed, we only need to define $H(x) \doteq L_{1} L_{2}|x|$, and then (8) follows with $L \doteq L_{1} L_{2}$. The last condition (9) may be ensured when designing the controller before taking into account the network, which corresponds to the first step of emulation design. In fact, the same assumption is made in [13], and it implies that $\dot{x}=f(x, \zeta)$ is $\mathcal{L}_{2}$ stable from $W$ to $H$. Any stabilisable and detectable linear time-invariant system does ensure this property. Examples of nonlinear systems that satisfy (9) can be found in e.g., [17], [18].

The main result of this section is stated below. It provides an explicit bound on $\tau_{\text {MATI }}$, which corresponds to the one in [14], that guarantees asymptotic stability of system (6).

Theorem 1: Consider system (6) and suppose that Assumption 2 holds. Then, if $\tau_{\text {MATI }}$ satisfies

$$
\tau_{\text {MATI }} \leq \begin{cases}\frac{1}{L r} \arctan \left(\frac{r(1-\lambda)}{2 \frac{\lambda}{1+\lambda}\left(\frac{\gamma}{L}-1\right)+1+\lambda}\right), & \gamma>L, \\ \frac{1}{L} \frac{1-\lambda}{1+\lambda}, & \gamma=L, \\ \frac{1}{L r} \operatorname{arctanh}\left(\frac{r(1-\lambda)}{2 \frac{\lambda}{1+\lambda}\left(\frac{\gamma}{L}-1\right)+1+\lambda}\right), & \gamma<L,\end{cases}
$$

where $r \doteq \sqrt{\left|\left(\frac{\gamma}{L}\right)^{2}-1\right|}$, then the set $\left\{\left(x, \zeta, \tau, \kappa, \tau_{s}, q\right)\right.$ : $x=0, \zeta=0\}$ is uniformly globally asymptotically stable (UGAS). That is, there exists $\beta \in \mathcal{K} \mathcal{L}$ such that, for any solution to $(6),|(x(t, j), \zeta(t, j))| \leq \beta(|(x(0,0), \zeta(0,0))|, t+j)$, for all $(t, j)$ in the solution's domain.

\section{SCHEDULING PROTOCOLS}

We present a general class of scheduling protocols that are implementable in WH under TDMA communications, and which satisfies properties (7) and (10).

\section{A. General class of scheduling protocols}

The following assumption serves to define the class of scheduling protocols.

Assumption 3:

(a) In each frequency division multiplexed channel, only one transmission between two field devices may be scheduled per timeslot.

(b) A field device cannot transmit and receive at the same time.

(c) All 15 available frequency channels may be used to schedule field devices.

(d) Superframes along frequency channels may have different periods (cf. Section III-B).

(e) Every field device needs to be scheduled to transmit in the superframe table at most in $\max _{h} T_{h}$ time units.

Parts (a)-(d) of Assumption 3 follow directly from [3].

Definition 1 (Class of TDMA scheduling protocols):

This class consists in all scheduling protocols for which their superframe tables are constructed under the guidelines of Assumption 3.

For this class of scheduling protocols, it is possible to show that the corresponding protocol equation is given by

$$
\zeta\left(t_{i}^{+}\right)=h\left(i, \zeta\left(t_{i}\right)\right)=\mathcal{H}(i) \zeta\left(t_{i}\right)
$$

where $\mathcal{H}(i) \doteq \operatorname{diag}\left\{\mathcal{H}^{y}(i), \mathcal{H}^{u}(i)\right\}$, and

$$
\begin{aligned}
& \mathcal{H}^{\star}(i) \doteq\left[\begin{array}{cc}
\Delta^{\star}(i) & 0 \\
I-\Delta^{\star}(i) & \Gamma^{\star}(i)
\end{array}\right], \\
& \Delta^{\star}(i) \doteq \operatorname{diag}\left\{\delta_{1}^{\star}(i), \ldots, \delta_{\ell_{\star}}^{\star}(i)\right\}, \\
& \Gamma^{\star}(i) \doteq\left[\begin{array}{cccc}
\gamma_{1}^{\star}(i) & & \\
1-\gamma_{1}^{\star}(i) & \gamma_{2}^{\star}(i) & & \\
\ddots & \ddots & \\
& 1-\gamma_{\ell_{\star}-1}^{\star}(i) & \gamma_{\ell_{\star}}^{\star}(i)
\end{array}\right],
\end{aligned}
$$

with $\star \in\{y, u\}$, and $\gamma_{\alpha}^{y}(i), \gamma_{\beta}^{u}(i), \delta_{\alpha}^{y}(i), \delta_{\beta}^{u}(i) \in\{0,1\}$, $\alpha=1, \ldots, \ell_{y}, \beta=1, \ldots, \ell_{u}$, which are defined differently according to the constructed superframe table. Later in this section, we provide specific scheduling protocols for which we give these definitions.

Define

$$
W(i, \zeta) \doteq \sqrt{\sum_{k=i}^{\infty}|\phi(k, i, \zeta)|^{2}}
$$

where $\phi(k, i, \zeta)$ denotes the solution of the auxiliary discretetime system $\zeta(i+1)=\mathcal{H}(i) \zeta(i)$ at time $k$ starting at time $i$ and initial condition $\zeta$, with $\mathcal{H}^{y}$ and $\mathcal{H}^{u}$ as in (12).

Theorem 2: Suppose Assumption 3 holds. Then,

(i) There exists $N \in \mathbb{Z}_{>0}$ such that the solution of the discrete-time system $\zeta(i+1)=\mathcal{H}(i) \zeta(i)$ converges to zero in $N$ steps. ( $N$ depends on the chosen superframe table, and thus is a function of $\ell_{y}$ and $\ell_{u}$.) 
(ii) System (11) satisfies (7) and (10) with Lyapunov function (13), $\underline{\alpha}_{W}(s)=s, \bar{\alpha}_{W}(s)=\sqrt{\frac{3^{N}-1}{2}} s$ for $s \geq 0$, $\lambda=\sqrt{\frac{3^{N}-3}{3^{N}-1}}$, and $L_{1}=\frac{3^{N}-1}{2}$.

Given that $N$ depends on the chosen superframe table, it is possible to decrease the bounds on $\lambda$ and $L$ by constructing scheduling protocols with small $N$. This, at the same time, would enlarge the MATI bound in Theorem 1, because it increases whenever $L$ and $\gamma$ decrease. In the remainder of this section, we provide three relevant scheduling protocols that can be implemented on WirelessHART and that belong to the previously presented class of protocols. We provide the value of $N$ for each one of these protocols and we show that, by exploiting multiple transmissions along the frequency channels, the MATI bound can be enlarged.

\section{B. Examples of scheduling protocols}

1) Simple Round Robin (S-RR): This scheduling protocol schedules the field devices in a round-robin manner [13], i.e., in a predetermined and cyclic manner. In other words, one frequency channel is used and the field devices communicate one after the other. In particular, we adopt the superframe table shown in Table I.

TABLE I

SUPERFRAME TABLE FOR THE S-RR PROTOCOL.

$$
\begin{array}{c|c:c:c:c:c:c:c} 
& t_{1} & t_{2} & \cdots & t_{\ell_{y}+1} & t_{\ell_{y}+2} & \cdots & t_{\ell_{y}+\ell_{u}+2} \\
\hline \mathrm{CH}_{1} & P \rightarrow D_{1}^{y} & D_{1}^{y} \rightarrow D_{2}^{y} & \cdots & D_{\ell_{y}}^{y} \rightarrow C & C \rightarrow D_{1}^{u} & \cdots & D_{\ell_{u}}^{u} \rightarrow P \\
\hline
\end{array}
$$

For this scheduling protocol, and enlightened by Table I, it is possible to show that (cf. (12)), $\delta_{\alpha}^{y}(i) \doteq 1-\mathbf{1}_{\mathcal{S}_{\alpha}^{y}}(i)$, $\delta_{\beta}^{u}(i) \doteq 1-\mathbf{1}_{\mathcal{S}_{\beta}^{u}}(i), \gamma_{\alpha}^{y}(i) \doteq 1-\mathbf{1}_{\overline{\mathcal{S}}_{\alpha}^{y}}(i)$, and $\gamma_{\beta}^{u}(i) \doteq 1-$ $\mathbf{1}_{\overline{\mathcal{S}}_{\beta}^{u}}(i)$, where

$\mathcal{S}_{\alpha}^{y} \doteq\left\{i \in \mathbb{N}: i=\alpha+\left(\ell_{y}+\ell_{u}+2\right) \sigma, \sigma \in \mathbb{Z}_{\geq 0}\right\}$,

$\mathcal{S}_{\beta}^{y} \doteq\left\{i \in \mathbb{N}: i=\beta+\ell_{y}+1+\left(\ell_{y}+\ell_{u}+2\right) \sigma, \sigma \in \mathbb{Z}_{\geq 0}\right\}$,

$\overline{\mathcal{S}}_{\alpha}^{y} \doteq\left\{i \in \mathbb{N}: i=\alpha+1+\left(\ell_{y}+\ell_{u}+2\right) \sigma, \sigma \in \mathbb{Z}_{\geq 0}\right\}$,

$\overline{\mathcal{S}}_{\beta}^{y} \doteq\left\{i \in \mathbb{N}: i=\beta+\ell_{y}+2+\left(\ell_{y}+\ell_{u}+2\right) \sigma, \sigma \in \mathbb{Z}_{\geq 0}\right\}$,

for $\alpha=1, \ldots, \ell_{y}$ and $\beta=1, \ldots, \ell_{u}$. Therefore, for this scheduling protocol, the parameter $N$ in Theorem 2 is given by $N \doteq N_{\text {s-RR }}=\max \left\{2 \ell_{y}+\ell_{u}+2,2 \ell_{u}+\ell_{y}+2\right\}$. Consequently, given Theorem 2 and the above, we have the following corollary.

Corollary 2: Consider system (11) for S-RR. Then, item (i) of Theorem 2 holds with $N \doteq N_{\text {S-RR }}=\max \left\{2 \ell_{y}+\ell_{u}+\right.$ $\left.2,2 \ell_{u}+\ell_{y}+2\right\}$; and conditions (7) and (10) are verified with $W$ as per (13), $\underline{\alpha}_{W}^{\text {S-RR }}(s)=s, \bar{\alpha}_{W}^{\text {S.RR }}(s)=\sqrt{\frac{3^{N_{S-R R}-1}}{2}} s$ for $s \geq 0, \lambda_{\mathrm{S}-\mathrm{RR}}=\sqrt{\frac{3^{N_{\mathrm{S}-\mathrm{R}}-3}}{3^{N_{\mathrm{S}-\mathrm{RR}}-1}}}$, and $L_{1, \mathrm{~S}-\mathrm{RR}}=\frac{3^{N_{\mathrm{S}-\mathrm{RR}}-1}}{2}$.

2) Frequency Division Duplex Round Robin (FDD-RR): This scheduling protocol establishes a full-duplex communication link that uses two different frequency channels for measurements and actuation operations. In particular, we consider the scheduling protocol given by Table II.

In this case, we have that $\delta_{\alpha}^{y}(i) \doteq 1-\mathbf{1}_{\mathcal{D}_{\alpha}^{y}}(i), \delta_{\beta}^{u}(i) \doteq$ $1-\mathbf{1}_{\mathcal{D}_{\beta}^{u}}(i), \gamma_{\alpha}^{y}(i) \doteq 1-\mathbf{1}_{\overline{\mathcal{D}}_{\alpha}^{y}}(i)$, and $\gamma_{\beta}^{u}(i) \stackrel{1^{\prime}}{\doteq} 1-\mathbf{1}_{\overline{\mathcal{D}}_{\beta}^{u}}(i)$,
TABLE II

SUPERFRAME TABLE FOR THE FDD-RR PROTOCOL.

\begin{tabular}{|c|c|}
\hline$\overline{\mathrm{CH}_{1}}$ & $P \rightarrow D_{1,}^{y_{1}} D_{1}^{y} \rightarrow D_{2,}^{y_{1}} \cdots D_{\ell_{y}-1}^{y} \rightarrow D_{\ell}^{y}$ \\
\hline$\overline{\mathrm{CH}_{2}}$ & 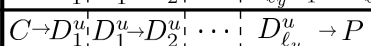 \\
\hline
\end{tabular}

where

$$
\begin{aligned}
& \mathcal{D}_{\alpha}^{y} \doteq\left\{i \in \mathbb{N}: i=\alpha+\left(\ell_{y}+1\right) \sigma, \sigma \in \mathbb{Z}_{\geq 0}\right\}, \\
& \mathcal{D}_{\beta}^{u} \doteq\left\{i \in \mathbb{N}: i=\beta+\left(\ell_{u}+1\right) \sigma, \sigma \in \mathbb{Z}_{\geq 0}\right\}, \\
& \overline{\mathcal{D}}_{\alpha}^{y} \doteq\left\{i \in \mathbb{N}: i=\alpha+1+\left(\ell_{y}+1\right) \sigma, \sigma \in \mathbb{Z}_{\geq 0}\right\}, \\
& \overline{\mathcal{D}}_{\beta}^{y} \doteq\left\{i \in \mathbb{N}: i=\beta+1+\left(\ell_{u}+1\right) \sigma, \sigma \in \mathbb{Z}_{\geq 0}\right\}
\end{aligned}
$$

for $\alpha=1, \ldots, \ell_{y}$ and $\beta=1, \ldots, \ell_{u}$. Thus, for this case the parameter $N$ in Theorem 2 is given by $N \doteq N_{\mathrm{FDD}-\mathrm{RR}}=$ $\max \left\{2 \ell_{y}+1,2 \ell_{u}+1\right\}$. Consequently, given Theorem 2 and the above, we have the following proposition.

Corollary 3: Consider system (11) for S-RR. Then, item (i) of Theorem 2 holds with $N \doteq N_{\text {FDD-RR }}=\max \left\{2 \ell_{y}+\right.$ $\left.1,2 \ell_{u}+1\right\}$; and conditions (7) and (10) are verified with $W$ as per (13), $\underline{\alpha}_{W}^{\text {FDD-RR }}(s)=s, \bar{\alpha}_{W}^{\mathrm{FDD}-\mathrm{RR}}(s)=\sqrt{\frac{3^{N_{\mathrm{FDD}}-\mathrm{RR}}-1}{2}} s$ for $s \geq 0, \lambda_{\mathrm{FDD}-\mathrm{RR}}=\sqrt{\frac{3^{N_{\mathrm{FDD}-\mathrm{RR}}-3}}{3^{N_{\mathrm{FDD}-\mathrm{RR}}-1}}}$, and $L_{1, \mathrm{FDD}-\mathrm{RR}}=\frac{3^{N_{\mathrm{FDD}-\mathrm{RR}}-1}}{2}$.

3) Wave Round Robin (W-RR): This scheduling protocol schedules devices in an interleaved manner. In particular, devices are scheduled according to Table III. In this manner, we are exploiting the multiple frequency channels of the network and it can be seen in Table III, that only two slots are required as the superframe length.

TABLE III

SUPERFRAME TABLE FOR THE W-RR PROTOCOL (FOR $\ell_{y}$ EVEN, $\ell_{u}$ ODD).

\begin{tabular}{c|c:c} 
& $t_{1}$ & $t_{2}$ \\
\hline $\mathrm{CH}_{1}$ & $P \rightarrow D_{1}^{y}$ & $D_{1}^{y} \rightarrow D_{2}^{y}$ \\
\hline $\mathrm{CH}_{2}$ & $D_{2}^{y} \rightarrow D_{3}^{y}$ & $D_{3}^{y} \rightarrow D_{4}^{y}$ \\
\hline$\vdots$ & $\vdots$ & $\vdots$ \\
\hline $\mathrm{CH}_{M_{y}-1}$ & $D_{\ell_{y}-2}^{y} \rightarrow D_{\ell_{y}-1}^{y}$ & $D_{\ell_{y}-1}^{y} \rightarrow D_{\ell_{y}}^{y}$ \\
\hline $\mathrm{CH}_{M_{y}}$ & $D_{\ell_{y}}^{y} \rightarrow C$ & \\
\hline $\mathrm{CH}_{M_{y}+1}$ & $C \rightarrow D_{1}^{u}$ & $D_{1}^{u} \rightarrow D_{2}^{u}$ \\
\hline $\mathrm{CH}_{M_{y}+2}$ & $D_{2}^{u} \rightarrow D_{3}^{u}$ & $D_{3}^{u} \rightarrow D_{4}^{u}$ \\
\hline$\vdots$ & $\vdots$ & $\vdots$ \\
\hline $\mathrm{CH}_{M_{y}+M_{u}-1}$ & $D_{\ell_{u}-3}^{u} \rightarrow D_{\ell_{u}-2}^{u}$ & $D_{\ell_{u}-2}^{u} \rightarrow D_{\ell_{u}-1}^{u}$ \\
\hline $\mathrm{CH}_{M_{y}+M_{u}}$ & $D_{\ell_{u}-1}^{y} \rightarrow D_{\ell_{u}}^{u}$ & $D_{\ell_{u}}^{u} \rightarrow P$ \\
\hline
\end{tabular}

Note that the number of channels used for the $y$-path (namely $M_{y}$ ), and the number of channels used for the $u$-path (namely $M_{u}$ ) satisfy $M_{y} \doteq \frac{\ell_{y}+2-\theta_{y}}{2}, \quad M_{u} \doteq \frac{\ell_{u}+2-\theta_{u}}{2}$, where $\theta_{y}, \theta_{u} \in\{0,1\}$. In particular, $\theta_{y}$ (resp. $\theta_{u}$ ) is 0 if $\ell_{y}$ (resp. $\ell_{u}$ ) is even, and 1 if $\ell_{y}$ (resp. $\ell_{u}$ ) is odd. Furthermore, $M_{y}$ and $M_{u}$ need to satisfy $M_{y}+M_{u} \leq 15$, which are the available channels in $\mathrm{WH}$.

For this case study, $\delta_{1+2 \alpha_{1}}^{y}(i) \doteq 1-\mathbf{1}_{\mathcal{W}}(i), \delta_{2+2 \alpha_{2}}^{y}(i) \doteq$ $\mathbf{1}_{\mathcal{W}}(i), \delta_{1+2 \beta_{1}}^{u}(i) \doteq 1-\mathbf{1}_{\mathcal{W}}(i), \delta_{2+2 \beta_{2}}^{u}(i) \doteq \mathbf{1}_{\mathcal{W}}(i), \gamma_{\alpha}^{y}(i) \doteq$ $1-\delta_{\alpha}^{y}(i)$, and $\gamma_{\beta}^{u}(i) \doteq 1-\delta_{\beta}^{u}(i)$, where

$$
\mathcal{W} \doteq\left\{i \in \mathbb{N}: i=1+2 \sigma, \sigma \in \mathbb{Z}_{\geq 0}\right\},
$$


for $\alpha_{1}=0,1, \ldots, \frac{\ell_{y}+\theta_{y}-2}{2}, \alpha_{2}=0,1, \ldots, \frac{\ell_{y}-\theta_{y}-2}{2}, \beta_{1}=$ $0,1, \ldots, \frac{\ell_{u}+\theta_{u}-2}{2}, \beta_{2}=0,1, \ldots, \frac{\ell_{u}-\theta_{u}-2}{2}, \alpha=1, \ldots, \ell_{y}$, and $\beta=1, \ldots, \ell_{u}$. Hence, for this case the parameter $N$ in Theorem 2 is given by $N \doteq N_{\text {W-RR }}=\max \left\{\ell_{y}+2, \ell_{u}+2\right\}$. Consequently, given Theorem 2 and the above, we have the following proposition.

Corollary 4: Consider system (11) for W-RR. Then, item (i) of Theorem 2 holds with $N \doteq N_{\text {W-RR }}=\max \left\{\ell_{y}+2, \ell_{u}+\right.$ $2\}$; and conditions (7) and (10) are verified with $W$ as per (13), $\underline{\alpha}_{W}^{\mathrm{W}-\mathrm{RR}}(s)=s, \bar{\alpha}_{W}^{\mathrm{W}-\mathrm{RR}}(s)=\sqrt{\frac{3^{N_{\mathrm{W}} \mathrm{RR}}-1}{2}} s$ for $s \geq 0$, $\lambda_{\mathrm{W}-\mathrm{RR}}=\sqrt{\frac{3^{N_{\mathrm{W}-\mathrm{RR}}-3}}{3^{N_{\mathrm{W}-\mathrm{RR}}-1}}}$, and $L_{1, \mathrm{~W}-\mathrm{RR}}=\frac{3^{N_{\mathrm{W}-\mathrm{RR}}-1}}{2}$.

It can be seen that $\lambda_{\mathrm{S}-\mathrm{RR}} \geq \lambda_{\mathrm{FDD}-\mathrm{RR}} \geq \lambda_{\mathrm{W}-\mathrm{RR}}$ and $L_{1, \mathrm{~S}-\mathrm{RR}} \geq$ $L_{1, \mathrm{FDD}-\mathrm{RR}} \geq L_{1, \mathrm{w}-\mathrm{RR}}$ for $\ell_{y}, \ell_{y}>0$. That is, exploiting multiple transmissions along frequency channels helps reducing $\lambda$ and $L$, thus enlarging the MATI bound. We will illustrate this with a numerical example.

\section{NumericAL EXAMPLE}

We illustrate our results in the stabilisation of the batch reactor in [13] over WirelessHART, for $\ell_{y}=2$ and $\ell_{u}=1$. We implement the three scheduling protocols of Section VI and compare the resulting MATI bounds given by Theorem 1 . Note that $N_{\text {S-RR }}=7, N_{\text {FDD-RR }}=5$ and $N_{\text {W-RR }}=4$. For the batch reactor, it can be seen that $L_{2}=35.51$. Moreover, given Propositions 1, 2 and 3, we have that $\lambda_{\mathrm{s}-\mathrm{RR}}=0.999, L_{1, \mathrm{~s}-\mathrm{RR}}=$ $1093, \lambda_{\mathrm{FDD}-\mathrm{RR}}=0.995, L_{1, \mathrm{FDD}-\mathrm{RR}}=121, \lambda_{\mathrm{W}-\mathrm{RR}}=0.987$, $L_{1, \mathrm{~W}-\mathrm{RR}}=40$. The gains $\gamma_{\mathrm{S}-\mathrm{RR}}, \gamma_{\mathrm{FDD}-\mathrm{RR}}, \gamma_{\mathrm{W}-\mathrm{RR}}$ can be obtained by MATLAB and are given by $\gamma_{\mathrm{S}-\mathrm{RR}}=4.19 \cdot 10^{4}, \gamma_{\mathrm{FDD}-\mathrm{RR}}=$ $4.63 \cdot 10^{3}, \gamma_{\mathrm{W}-\mathrm{RR}}=1.53 \cdot 10^{3}$. With the above we obtain the bounds in Table IV, in view of Theorem 1. We also estimate the actual bound observed in simulations, i.e., we simulate the NCS and we compute $\tau_{\text {est }}$ such that the system remains stable for $\left(0, \tau_{\text {est }}\right]$. It can be seen that the bounds from Theorem 1 are quite conservative. A first attempt to improve this bound is to exploit, in the proof of Theorem 2, the fact that $|\mathcal{H}(i) \mathcal{H}(j)| \leq|\mathcal{H}(i)||\mathcal{H}(j)| \leq 3$ for all $i, j \in \mathbb{N}$. By doing so, we are able to obtain smaller values for $\lambda$ and $L_{1}$, which lead to significantly larger MATI bounds, as seen on the last line of Table IV. This direction will be carefully investigated in future work.

\section{TABLE IV}

COMPARISON OF MATIS FOR THE BATCH REACTOR

\begin{tabular}{||l|c|c|c||}
\hline & S-RR & FDD-RR & W-RR \\
\hline $\begin{array}{l}\text { MATI bound via } \\
\text { Theo. 1 (in [ms]) }\end{array}$ & $0.057 \cdot 10^{-4}$ & $4.65 \cdot 10^{-4}$ & $42.9 \cdot 10^{-4}$ \\
\hline $\begin{array}{l}\text { Simulation } \\
\text { bound (in [ms]) }\end{array}$ & 12 & 22 & 38 \\
\hline $\begin{array}{l}\text { Less conservative } \\
\text { bound (in [ms]) }\end{array}$ & 0.14 & 0.23 & 0.41 \\
\hline
\end{tabular}

It can be seen from Table IV, that implementing the WRR scheduling protocol results in better MATI bounds in comparison to S-RR and FDD-RR.

\section{CONCLUSIONS}

A new model that describes NCSs implemented on WirelessHART is needed in order to capture most functionalities of the network under reasonable assumptions. Such a model is developed, which we then use to obtain stability results in terms of MATI bounds. We also studied scheduling in WH networks by presenting a general class of TDMA scheduling protocols implementable under our model. We proved that such class satisfies the underlying stability assumptions of the emulation result. Moreover, we presented three relevant scheduling protocols that belong to that class and we showed that MATI bounds can actually be improved if you exploit simultaneous transmissions over multiple radio frequencies of the network. Future work will focus on studying WHNCSs under different topologies and communication constraints like packet dropouts.

\section{REFERENCES}

[1] J. Hespanha, P. Naghshtabrizi, and Y. Xu, "A survey of recent results in networked control systems," Proceedings of the IEEE, vol. 95, no. 1, pp. 138-162, January 2007.

[2] W. Heemels and N. Van De Wouw, "Stability and stabilization of networked control systems," in Networked Control Systems. Springer, 2010, pp. 203-253.

[3] "HART Communication Protocol," http://en.hartcomm.org/.

[4] H. Zhang, P. Soldati, and M. Johansson, "Time-and channel-efficient link scheduling for convergecast in WirelessHART networks," in Proceedings of the 13th IEEE International Conference on Communication Technology (ICCT), 2011.

[5] — - "Performance bounds and latency-optimal scheduling for convergecast in WirelessHART networks," IEEE Transactions on Wireless Communications, vol. 12, no. 6, pp. 2688-2696, 2013.

[6] Z. Zou, B. Demirel, and M. Johansson, "Minimum-energy packet forwarding policies for guaranteed LQG performance in wireless control systems," in Proceedings of the 51st Conference on Decision and Control, 2012.

[7] B. Demirel, Z. Zou, P. Soldati, and M. Johansson, "Modular design of jointly optimal controllers and forwarding policies for wireless control," IEEE Transactions on Automatic Control, vol. 59, no. 12, pp. 3252-3265, 2014.

[8] R. Alur, A. d'Innocenzo, K. H. Johansson, G. J. Pappas, and G. Weiss, "Compositional modeling and analysis of multi-hop control networks," IEEE Transactions on Automatic control, vol. 56, no. 10, pp. 23452357, 2011.

[9] A. W. Al-Dabbagh and T. Chen, "A fixed structure topology for wireless networked control systems," in Proceedings of the 55th Conference on Decision and Control, Las Vegas, U.S.A., 2016, pp. 3450-3455.

[10] R. Goebel, R. Sanfelice, and A. Teel, Hybrid Dynamical Systems: modeling, stability, and robustness. Princeton University Press, 2012.

[11] W. Wang, D. Nešić, and R. Postoyan, "Emulation-based stabilization of networked control systems implemented on FlexRay," Automatica, vol. 59, pp. 73-83, 2015 .

[12] W. Wang, D. Nešić, and R. Postoyan, "Observer design for networked control systems with flexray," Automatica, vol. 82, pp. 42-48, 2017.

[13] D. Nešić and A. Teel, "Input-output stability properties of networked control systems," IEEE Transactions on Automatic Control, vol. 49, no. 10, pp. 1650-1667, 2004.

[14] D. Carnevale, A. Teel, and D. Nešić, "A Lyapunov proof of an improved maximum allowable transfer interval for networked control systems," IEEE Transactions on Automatic Control, vol. 52, no. 5, p. 892, 2007.

[15] G. Walsh, O. Beldiman, and L. Bushnell, "Asymptotic behavior of nonlinear networked control systems," IEEE Transactions on Automatic Control, vol. 46, no. 7, pp. 1093-1097, jul 2001.

[16] A. Maass, D. Nešić, and P. Dower, "A hybrid model of networked control systems implemented on WirelessHART networks under source routing configuration," in Australian Control Conference, Newcastle, Australia, 2016.

[17] D. Nešić, A. R. Teel, and D. Carnevale, "Explicit computation of the sampling period in emulation of controllers for nonlinear sampled-data systems," IEEE Transactions on Automatic Control, vol. 54, no. 3, pp. 619-624, 2009.

[18] M. Abdelrahim, R. Postoyan, J. Daafouz, and D. Nešić, "Stabilization of nonlinear systems using event-triggered output feedback controllers," IEEE Transactions on Automatic Control, vol. 61, no. 9, pp. 2682-2687, 2016. 


\section{University Library}

\section{- M M I N E R VA A gateway to Melbourne's research publications}

Minerva Access is the Institutional Repository of The University of Melbourne

Author/s:

Maass, Al;Nesic, D;Postoyan, R;Dower, PM;S Varma, V

Title:

Emulation-based stabilisation of networked control systems over WirelessHART

Date:

2017

Citation:

Maass, A. I., Nesic, D., Postoyan, R., Dower, P. M. \& S Varma, V. (2017). Emulation-based

stabilisation of networked control systems over WirelessHART. Proceedings of the IEEE 56th Annual Conference on Decision and Control (CDC 2017), 2018-January, IEEE Press. https:// doi.org/10.1109/CDC.2017.8264657.

Persistent Link:

http://hdl.handle.net/11343/251424 